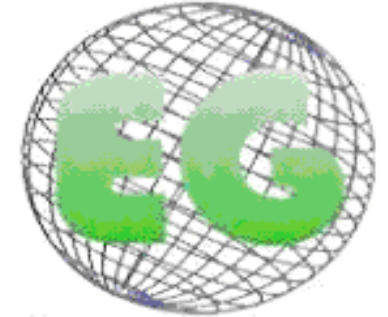

ISSN 1695-6141

$\mathrm{N}^{\circ} 21$
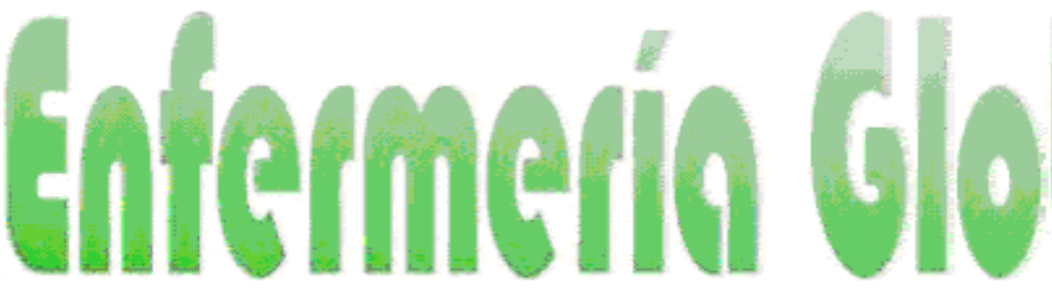

Revista electrónica trimestral de Enfermería

Enero 2011

www.um.es/egloball

\title{
EVOLUCIÓN DE LOS PARTOS MÚLTIPLES EN LA REGIÓN DE MURCIA DESDE 1.996 HASTA 2.008: IMPLICACIONES PSICOSOCIALES
}

\author{
EVOLUTION OF MULTIPLE DELIVERIES IN THE REGION OF MURCIA FROM 1996 TO
} 2008: PSYCHOSOCIAL IMPLICATIONS

*Tornero Gómez, MJ., Sánchez Romera, JF., Colodro Conde, L., Ordoñana Martín, JR.

*Departamento de Anatomía Humana y Psicobiología. Universidad de Murcia.

Palabras clave: embarazo múltiple, parto múltiple, gemelos, tasa de gemelos.

Keywords: Multiple Pregnancy, Multiple Delivery, Twins, Twin Rates.

\section{RESUMEN}

Los partos múltiples conllevan una serie de implicaciones psicosociales que afectan tanto a los padres como a los sistemas de atención sanitaria y social. La posibilidad de que se produzcan complicaciones se incrementa en el caso de un embarazo múltiple, así como las necesidades de atención y cuidados tanto para los bebés como para los padres. Disponer de información acerca de la evolución de este tipo de embarazos resulta, por tanto, de gran relevancia para identificar necesidades de salud en la población. Objetivos: Este trabajo tiene como principales objetivos presentar la evolución de los partos múltiples entre los años 1996 y 2008, tanto en la Región de Murcia como en España, examinar estos datos en función de la edad de la madre en el momento del nacimiento y analizar las posibles repercusiones de esta evolución. Método: Para ello se ha utilizado la información disponible en las bases de datos del Instituto Nacional de Estadística y del Centro Regional de Estadística de Murcia. Resultados: En los últimos años se ha producido un importante aumento en el número de partos múltiples tanto a nivel nacional como en las distintas comunidades autónomas, la Región de Murcia entre ellas. La tasa de partos gemelares o de orden mayor ha pasado a ser de 13.1\%o en 1996 a 18.4\%o en 2008 en Murcia (12.7\% a 19.4\%o en España). El mayor incremento se ha producido en los grupos de madres a partir de los 30 años. Conclusiones: Esta situación presenta implicaciones importantes para el sistema de salud en su conjunto y para las familias. Estas necesitan conseguir una adaptación en la que puede colaborar de forma decisiva el profesional sanitario proporcionando una información y orientación adecuadas. 


\section{ABSTRACT}

Multiple delivery carries a series of psychosocial implications which concern both the parents and the social and health care systems. The possibility of complications increases in multiple pregnancies, as well as the needs for attention and care for both, the babies and the parents. Therefore, information about the evolution of this kind of pregnancies is very important to identify people's health needs. Objectives: The main objective of this research is to show the evolution of multiple deliveries in Spain, and in the Murcia Region between 1996 and 2008. Additional objectives are to examine those data according to mother's age at maternity, and to analyze possible consequences of such evolution. Method: Information has been obtained from the National Institute of Statistics and the Murcia Regional Centre of Statistics databases. Results: Multiple births rate has changed form $13.1 \%$ in 1996 to $18.4 \%$ in 2008 in Murcia (12.7\%o to $19.4 \%$ in Spain). The biggest increase has taken place in mothers older than 30 years old. Conclusion: This situation presents important implications for the families and for the health system as a whole. Families need to reach an adaptation and health workers might collaborate giving adequate information and orientation.

\section{INTRODUCCIÓN}

En los últimos años, el número de partos múltiples en España se ha incrementado de forma constante. ${ }^{1}$ Este hecho ha ido acompañado, de forma paralela, por un aumento general del número de nacimientos en nuestro país; si bien la magnitud del incremento ha sido menor en este último caso. Una posible explicación de este aumento en el número de nacimientos es el fenómeno de la inmigración, pero ésta no es considerada la principal causa del aumento de partos múltiples. El incremento de los embarazos múltiples parece deberse a varios factores, entre los que destacan el incremento de la edad a la maternidad y, principalmente, la creciente utilización de técnicas de reproducción asistida. ${ }^{1}$ Según apuntan Bruna Catalán y cols., ${ }^{2}$ España muestra una incidencia muy elevada de partos múltiples tras la utilización de técnicas de reproducción asistida. Así, entre los años 1.980 y 2.002, se incrementaron notablemente el número de unidades de reproducción y el número de ciclos de tratamientos de reproducción asistida que se llevaron a cabo, lo que contribuyó, en gran medida, al elevado crecimiento de los partos gemelares y triples. La tasa de estos últimos, por ejemplo, se multiplicó por siete en este periodo (11 cada 100.000 partos en el año 1.980; 76 cada 100.000 partos en el 2.002), y llegó a ser casi tres veces superior a la registrada en Europa (4,1\%o frente al 1,5\% europeo). ${ }^{2}$ Además, los dos factores mencionados están relacionados entre sí puesto que, a mayor edad de la mujer, mayor dificultad para quedar embarazada y, por tanto, mayor probabilidad de que se someta a un tratamiento de fertilidad.

Los embarazos múltiples implican un gran número de exigencias psicosociales, influyendo no sólo sobre los padres y el entorno familiar cercano, sino también sobre los servicios socio-sanitarios que atienden a las personas que se encuentran en esta situación. Una de las principales funciones de los profesionales sanitarios debe ser la de ayudar a las familias a prepararse para conseguir gestionar con éxito las demandas que conllevan un embarazo múltiple y los primeros años tras el nacimiento de los niños ${ }^{3}$. Estos profesionales, donde podemos incluir a los médicos de familia, las enfermeras, los médicos obstetras, o las matronas, no han recibido, habitualmente, información o entrenamiento específico para tratar con las necesidades especiales que presentan las familias con dos o más niños naciendo al mismo tiempo. ${ }^{4}$

Tener un hijo conlleva grandes repercusiones sobre los padres, tanto a nivel económico, como social y psicológico. Estas repercusiones se ven incrementadas de forma drástica en el caso de un parto múltiple. Los padres que se encuentran en esta situación precisan de personal que los aconseje y de un gran apoyo de su entorno. 
A nivel económico, los embarazos múltiples requieren una cantidad de recursos de los servicios sanitarios mucho mayor a la requerida por un embarazo simple: mayores costes en las ecografías, en los cuidados prenatales, en los cuidados postnatales, en el parto, mayor utilización de la unidad de cuidados intensivos neonatal, etc., especialmente si los niños son prematuros o tienen complejas necesidades de salud ${ }^{4}$. En este sentido, se pueden diferenciar los costes destinados a proporcionar los recursos necesarios para la madre de los requeridos para los niños. Los costes asociados con la madre provienen de la morbilidad asociada al embarazo: trastornos hipertensivos, diabetes, desprendimiento de placenta, etc.; trastornos que incrementan el riesgo de cesárea, el tiempo de ingreso y el coste del tratamiento de estas mujeres. En el caso de los recién nacidos, los embarazos múltiples tienen asociado un $46,5 \%$ más de morbilidad debido al riesgo de que el parto se adelante. ${ }^{5}$

Aunque el desarrollo de la mayoría de los niños nacidos por parto múltiple está dentro del rango normal, tanto por razones médicas como ambientales, estos niños se enfrentan con un mayor riesgo de discapacidad a largo plazo 6, 7. Existe una incidencia mayor de anormalidades congénitas en asociación con los partos gemelares, lo cual, asociado con el parto prematuro y el bajo peso al nacer, contribuye a que la discapacidad sea más común entre estos niños. ${ }^{4}$

Pero además, el impacto económico sobre las familias que tienen gemelos, trillizos o un embarazo de mayor orden es considerable, debido a la posible pérdida de ingresos por salida de la madre del mercado laboral, y el enorme incremento de los gastos. ${ }^{3}$ Entre los nuevos costes pueden encontrarse desde la necesidad de comprar carritos, cunas, sillas... para gemelos o trillizos hasta incluso un coche más grande o una nueva casa, o la adaptación de la que ya tienen. Una pequeña minoría de las mujeres que esperan gemelos pueden seguir trabajando hasta poco antes del parto, pero la mayoría se ven obligadas a abandonar su puesto de trabajo anticipadamente, lo que puede conllevar además una pérdida de su estatus social y de un factor de protección importante para su salud mental. La mayor parte de las familias que esperan gemelos, a pesar de ver disminuidos fuertemente sus ingresos, no pueden optar a un subsidio o un apoyo financiero extra. Los padres que se encuentran en esta situación deberían ser alentados a explorar los recursos de apoyo material en su comunidad, y a solicitar el asesoramiento de otros padres que han pasado por su situación con el fin de adquirir los suministros necesarios y no hacer compras inapropiadas ${ }^{3}$.

A lo ya mencionado se unen los necesarios cambios en la vida social y familiar que pueden incluir limitación de los contactos sociales, cambios en las redes de apoyo, o la necesidad de adaptación y reorganización de horarios laborales y de actividades de ocio.

Por otra parte, tanto los embarazos múltiples, como los tratamientos de fertilidad, tienen importantes implicaciones psicológicas; por ello resulta tan recomendable el asesoramiento para las madres primerizas con un embarazo múltiple, especialmente para aquéllas que hayan tenido una historia de infertilidad previa. ${ }^{8}$ Estas implicaciones pueden observarse en tres dominios que, con mucha frecuencia, presentan relaciones y solapamientos entre sí: el deseo de ser padres, la ansiedad ante el embarazo y la maternidad/paternidad y la posibilidad de aparición de sintomatología depresiva. 


\section{Deseo de ser padres}

Generalmente las mujeres que se someten a un tratamiento de fertilidad prefieren tener un embarazo múltiple, especialmente aquéllas pacientes que no tienen hijos previamente. ${ }^{9} \mathrm{EI}$ deseo de un parto múltiple suele ser igual en las mujeres que en los hombres, aunque éstas suelen ser más conscientes de los riesgos que conlleva que sus parejas. ${ }^{10}$ Paralelamente las mujeres parecen asumir más fácilmente los riesgos asociados a un embarazo de este tipo, demostrando, en este caso, una menor aversión al riesgo que los varones.

La noticia de un embarazo múltiple puede ser recibida por los padres de manera muy diferente. Puede darse el caso de que el deseo de tener a sus hijos haga que no valoren de forma realista los posibles riesgos de este tipo de embarazo, ${ }^{10}$ o que el impacto de esta noticia cause altos niveles de ansiedad al considerar los posibles riesgos asociados, las consecuencias económicas, o las repercusiones sobre los distintos miembros de la familia. Del mismo modo pueden surgir diferentes emociones o estados negativos tras el parto, especialmente durante los primeros meses de vida de los hijos. En ambos casos las consecuencias negativas pueden disminuir con la información y la orientación adecuada que se dé a los padres.

\section{Ansiedad}

Se ha apuntado que algunas de las consecuencias negativas que puede tener el embarazo múltiple pueden desencadenar la aparición de síntomas ansiosos o depresivos, pudiendo afectar hasta a un $25 \%$ de los padres que se encuentran en esta situación. ${ }^{11}$ En realidad, la ansiedad experimentada por una madre acerca de la salud y el bienestar de sus hijos es observable en todos los embarazos, pero se intensifica especialmente en el caso de embarazos múltiples. Según el estudio de Fisher y Stocky, ${ }^{12}$ en el caso de haber tenido una historia previa de infertilidad, las consecuencias pueden ser aún más negativas, debido a lo idealizado que los padres pueden tener el embarazo, y a la subestimación de los riesgos reales que puede conllevar. El problema surge cuando estos padres, teniendo miedo acerca del bienestar de sus hijos y de su capacidad para cuidarlos, creen no tener derecho a expresar esta ambivalencia después del tratamiento que han seguido. ${ }^{12}$

Que exista un mayor riesgo de presentar síntomas ansiosos no implica que éstos siempre se presenten, pero sí que puede aparecer un "mayor nivel de estrés en la mujer que se somete a un tratamiento de fertilidad, siendo mayor su preocupación acerca de su capacidad para ser buena madre". ${ }^{8}$ Esta afirmación, sin embargo, no es apoyada por otros datos que concluyen que existe una mayor ansiedad acerca del cuidado de los niños en el caso de mujeres que tienen un embarazo múltiple de forma natural. ${ }^{13}$

\section{Depresión}

Igualmente los padres de bebés nacidos en un parto múltiple pueden presentar más síntomas depresivos durante el período prenatal y postnatal. ${ }^{11}$ La estimación de depresión postparto en mujeres adultas (tanto para embarazos simples como múltiples) se sitúa entre un 7 y un $16 \%$, siendo severa en un $2-3 \%$ de los casos. ${ }^{11,14}$ En el caso de partos múltiples, la depresión postparto puede afectar hasta a un 25-30\% de mujeres con un parto doble, y a más de un $40 \%$ de mujeres con parto triple, durante el primer año. ${ }^{15,16}$

Algunos de los factores que pueden influir en la aparición de estos síntomas son: la alta prevalencia de partos prematuros, las numerosas y constantes necesidades que tienen los 
hijos recién nacidos, la presión por satisfacer las expectativas individualizadas de los padres, un apoyo emocional insuficiente, la privación del sueño, y el aislamiento social. ${ }^{11}$

Aunque los tratamientos de fertilidad y los embarazos múltiples pueden ocasionalmente tener un efecto negativo sobre los padres, estos efectos pueden reducirse siempre que existan factores de protección social, como puede ser la estabilidad económica, y cualidades positivas en los padres a la hora de afrontar la nueva situación: adecuada resolución de problemas, buena relación entre los miembros de la pareja, o deseo compartido de paternidad.

\section{Conclusión y objetivos}

En conjunto, como se puede observar, el embarazo y parto múltiple presenta importantes consecuencias de diversa índole con repercusiones en la vida personal y de pareja, pero también en el sistema sanitario, que tiene que hacer frente a un incremento de costes y de atención, y en el profesional que debe dar respuesta a nuevas necesidades para las que no está preparado específicamente.

Este trabajo tiene como objetivo presentar la evolución de los partos múltiples durante los últimos años, tanto en la Región de Murcia como en España. En base a esta información trata de analizar cuáles pueden ser las posibles repercusiones en el marco de la atención socio-sanitaria, y qué respuestas podrían ofrecerse ante las nuevas necesidades generadas en las personas y familias involucradas en esta situación.

\section{MÉTODO}

Los datos utilizados en este informe han sido obtenidos a partir de las bases de datos del Instituto Nacional de Estadística ${ }^{18}$ y del Centro Regional de Estadística de Murcia. ${ }^{19}$ De las páginas web mencionadas se han recogido los datos referidos a nacimientos y partos (sencillos y múltiples) registrados en la Región de Murcia y en España entre los años 1996 y 2008. Adicionalmente, se han recogido los datos sobre partos en función de la edad de la madre. Dichos datos han sido elaborados, posteriormente, para realizar las tablas y gráficas presentadas.

\section{RESULTADOS}

\section{Nacimientos y partos múltiples en la Región de Murcia (1996-2008)}

En la Figura 1 se presenta el número total de partos múltiples en la Región de Murcia durante los últimos años (1996-2008) junto con la evolución que ha seguido el total de nacimientos en la Región durante el mismo período de tiempo. En lo referente a estos últimos, como se puede observar, la evolución ha sido positiva. En los años en los que nos estamos centrando, los nacimientos han aumentado de forma sustancial. Al final de la década de los 90 el número de nacimientos fue incrementándose de forma lenta, pero a partir del año 2000 el crecimiento es mucho más rápido. Este incremento, registrado también en España (Tabla 1), se supone producido, sobre todo, por la aportación de la llegada masiva de inmigrantes en este periodo. 
Figura 1: Partos múltiples y nacimientos en la Región de Murcia (1.996-2.008)

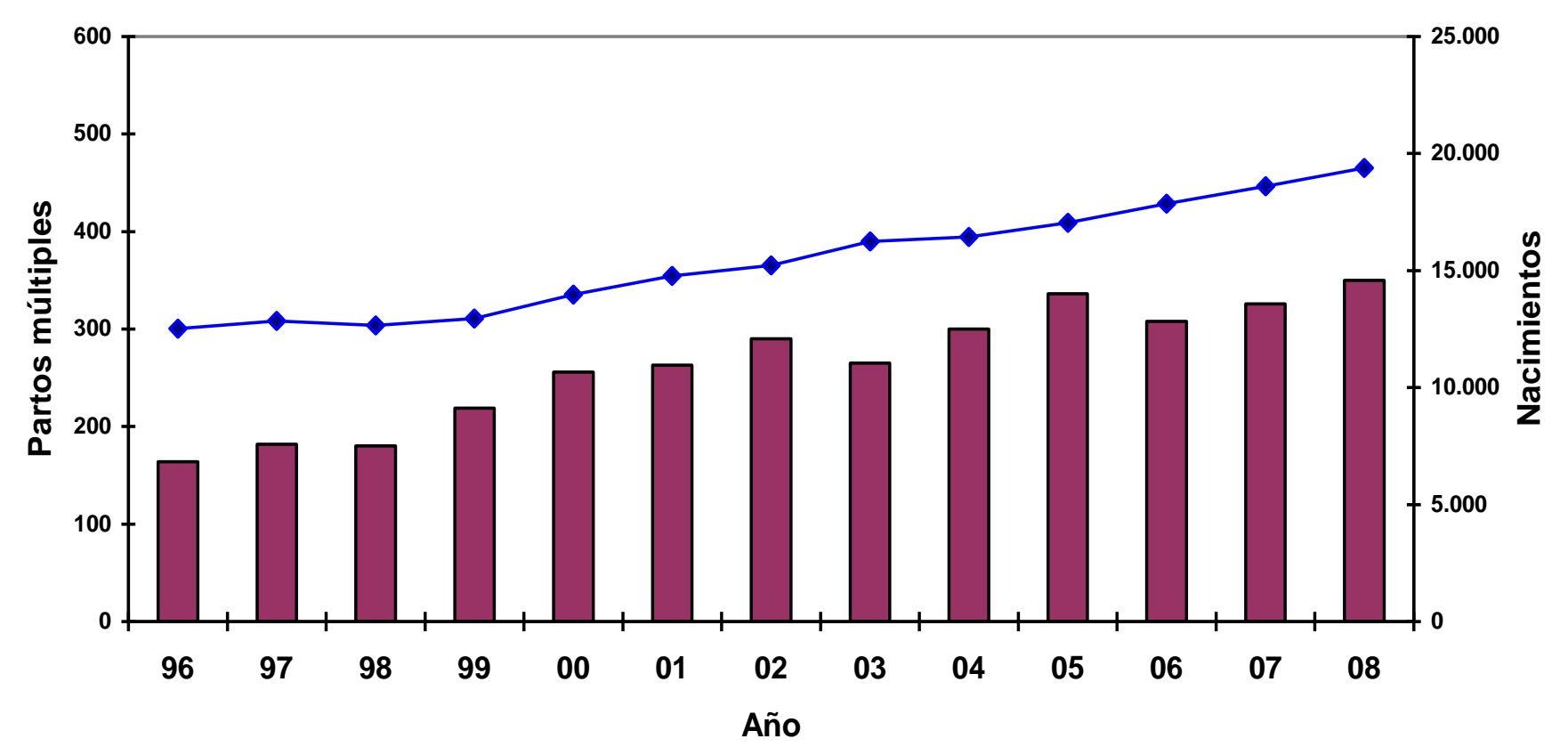

$\rightleftarrows$ Partos múltiples $\longrightarrow$ Nacimientos

Por lo que respecta a los partos múltiples, podemos observar en la gráfica cómo el año en el cual se registraron menos partos múltiples fue 1996, con un total de 164 partos. A partir de aquí se produjo un incremento sostenido hasta 2005 (336 partos, lo que representa un incremento de más del 100\%) y lo que parece una cierta estabilización posterior. Esta evolución coincide además con la producida a nivel general en España, como podemos ver en la Tabla 1.

Tabla 1: Partos en España y en la Región de Murcia según multiplicidad (1.996-2.008)

\begin{tabular}{|c|c|c|c|c|c|}
\hline \multirow{2}{*}{ AÑO: } & LUGAR: & $\begin{array}{c}\text { PARTOS } \\
\text { SENCILLOS: }\end{array}$ & $\begin{array}{c}\text { PARTOS } \\
\text { DOBLES: }\end{array}$ & $\begin{array}{c}\text { PARTOS } \\
\text { TRIPLES: }\end{array}$ & $\begin{array}{c}\text { PARTOS } \\
\text { CUÁDRUPLES } \\
\text { O MÁS: }\end{array}$ \\
\hline \multirow{3}{*}{1.996} & ESPAÑA & 354.729 & 4.433 & 139 & 8 \\
\cline { 2 - 6 } & MURCIA & 12.361 & 154 & 10 & 0 \\
\hline \multirow{3}{*}{1.997} & ESPAÑA & 360.743 & 4.649 & 165 & 7 \\
\cline { 2 - 6 } & MURCIA & 12.666 & 175 & 6 & 1 \\
\hline \multirow{3}{*}{1.998} & ESPAÑA & 356.378 & 4.820 & 189 & 6 \\
\cline { 2 - 6 } & MURCIA & 12.482 & 173 & 7 & 0 \\
\hline \multirow{2}{*}{2.000} & ESPAÑA & 370.003 & 5.457 & 216 & 7 \\
\cline { 2 - 6 } & MURCIA & 12.726 & 216 & 3 & 0 \\
\cline { 2 - 6 } & ESPAÑA & 386.336 & 5.975 & 267 & 6 \\
\hline \multirow{2}{*}{2.001} & MURCIA & 13.726 & 253 & 3 & 0 \\
\cline { 2 - 6 } & ESPAÑA & 394.507 & 6.234 & 306 & 7 \\
\cline { 2 - 6 } & MURCIA & 14.508 & 254 & 9 & 0 \\
\hline \multirow{2}{*}{2.002} & ESPAÑA & 405.512 & 6.929 & 302 & 10 \\
\cline { 2 - 6 } & MURCIA & 14.939 & 278 & 12 & 0 \\
\hline
\end{tabular}




\begin{tabular}{|c|c|c|c|c|c|}
\hline \multirow{2}{*}{2.003} & ESPAÑA & 427.457 & 7.499 & 300 & 5 \\
\cline { 2 - 6 } & MURCIA & 15.975 & 261 & 4 & 0 \\
\hline \multirow{2}{*}{2.004} & ESPAÑA & 439.806 & 7.712 & 265 & 1 \\
\cline { 2 - 6 } & MURCIA & 16.131 & 294 & 6 & 0 \\
\hline \multirow{2}{*}{2.005} & ESPAÑA & 450.831 & 8.225 & 208 & 1 \\
\cline { 2 - 6 } & MURCIA & 16.708 & 329 & 7 & 0 \\
\hline \multirow{2}{*}{2.006} & ESPAÑA & 466.913 & 8.510 & 212 & 0 \\
\cline { 2 - 6 } & MURCIA & 17.547 & 303 & 5 & 0 \\
\hline \multirow{2}{*}{2.007} & ESPAÑA & 474.833 & 9.254 & 231 & 1 \\
\cline { 2 - 6 } & MURCIA & 18.016 & 320 & 6 & 0 \\
\hline \multirow{2}{*}{2.008} & ESPAÑA & 501.334 & 9.696 & 213 & 4 \\
\cline { 2 - 6 } & MURCIA & 18.747 & 349 & 1 & 0 \\
\hline
\end{tabular}

\section{Tasa de partos múltiples en la Región de Murcia y en España (1.996-2.008)}

La Figura 2 nos proporciona información comparativa de la tasa de partos múltiples en Murcia y en España entre los años 1996 y 2008. La evolución es creciente en ambos casos, con alguna excepción en los datos de Murcia. A nivel nacional, la tasa se incrementa de forma gradual y muy regular, mientras que a nivel regional, observamos mayor irregularidad con incrementos y también descensos más pronunciados.

Figura 2: Tasas de partos múltiples en la Región de Murcia y en España (1.996-2.008)

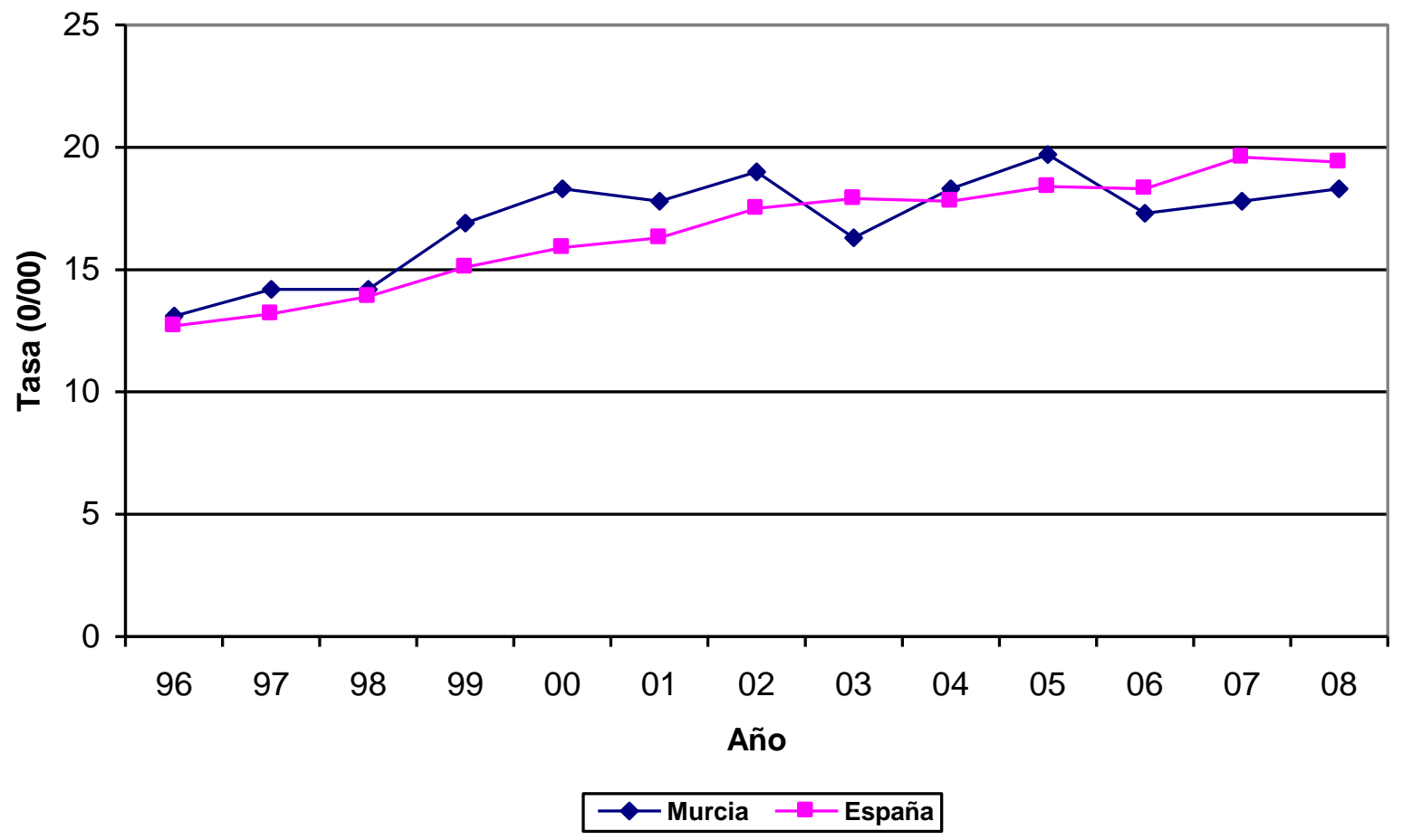

La Figura también nos muestra la diferencia existente entre la tasa obtenida en cada uno de los años en Murcia con respecto a España, siendo normalmente superior la de Murcia, independientemente de su magnitud. Por ejemplo, en el año 1998 apenas hubo diferencia 
entre la tasa a nivel nacional y la tasa a nivel regional, mientras que en el año 2000 la diferencia fue de más de 2 puntos.

\section{Tasa de partos múltiples en función del grupo de edad de la madre en la Región de Murcia y en España (1.996-2.008)}

En las Figuras 3 y 4 encontramos las tasas de partos múltiples en función del grupo de edad de la madre. La Figura 3 contiene los datos referidos a España, y la Figura 4 contiene los datos de la Región de Murcia.

En la distribución de datos correspondiente al total nacional podemos observar, como cabía esperar, cómo las mayores tasas de partos múltiples corresponden a mujeres con más de 34 años, seguidas por el grupo de edad comprendido entre 30 y 34 años. También resulta relevante cómo las tasas de estos dos grupos son las que se han ido incrementando en la última década, mientras en los grupos de menor edad las tasas se han mantenido relativamente constantes. Por otra parte, podemos observar también cómo el incremento en las tasas de partos múltiples en mujeres de más de 30 años se mantiene hasta el año 2.002, pero a partir de entonces se produce una estabilización en las mismas.

Figura 3: Tasas de partos múltiples según grupo de edad de la madre en España (1.996-2.008)

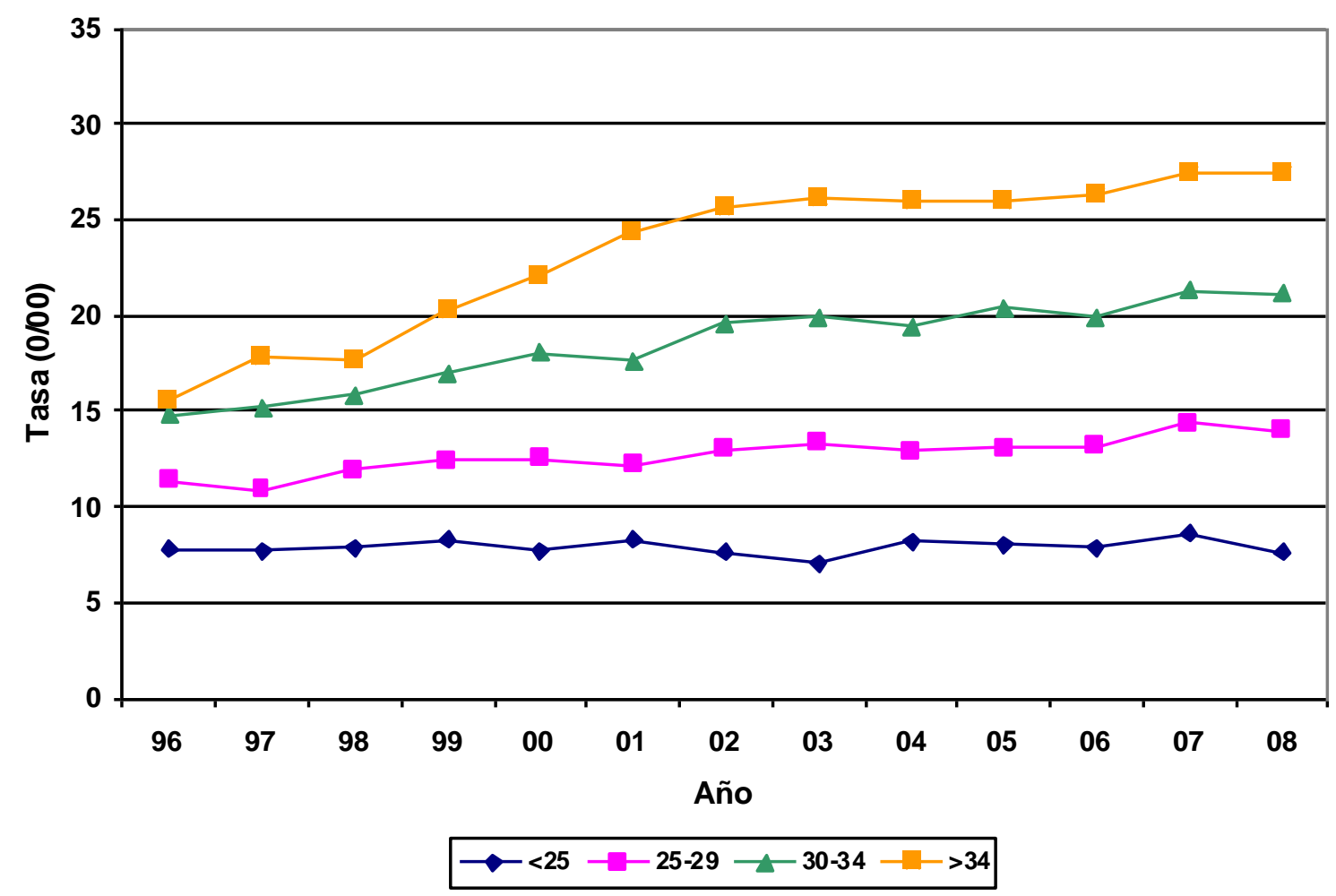


Figura 4: Tasas de partos múltiples según grupo de edad de la madre en la Región de Murcia (1.996-

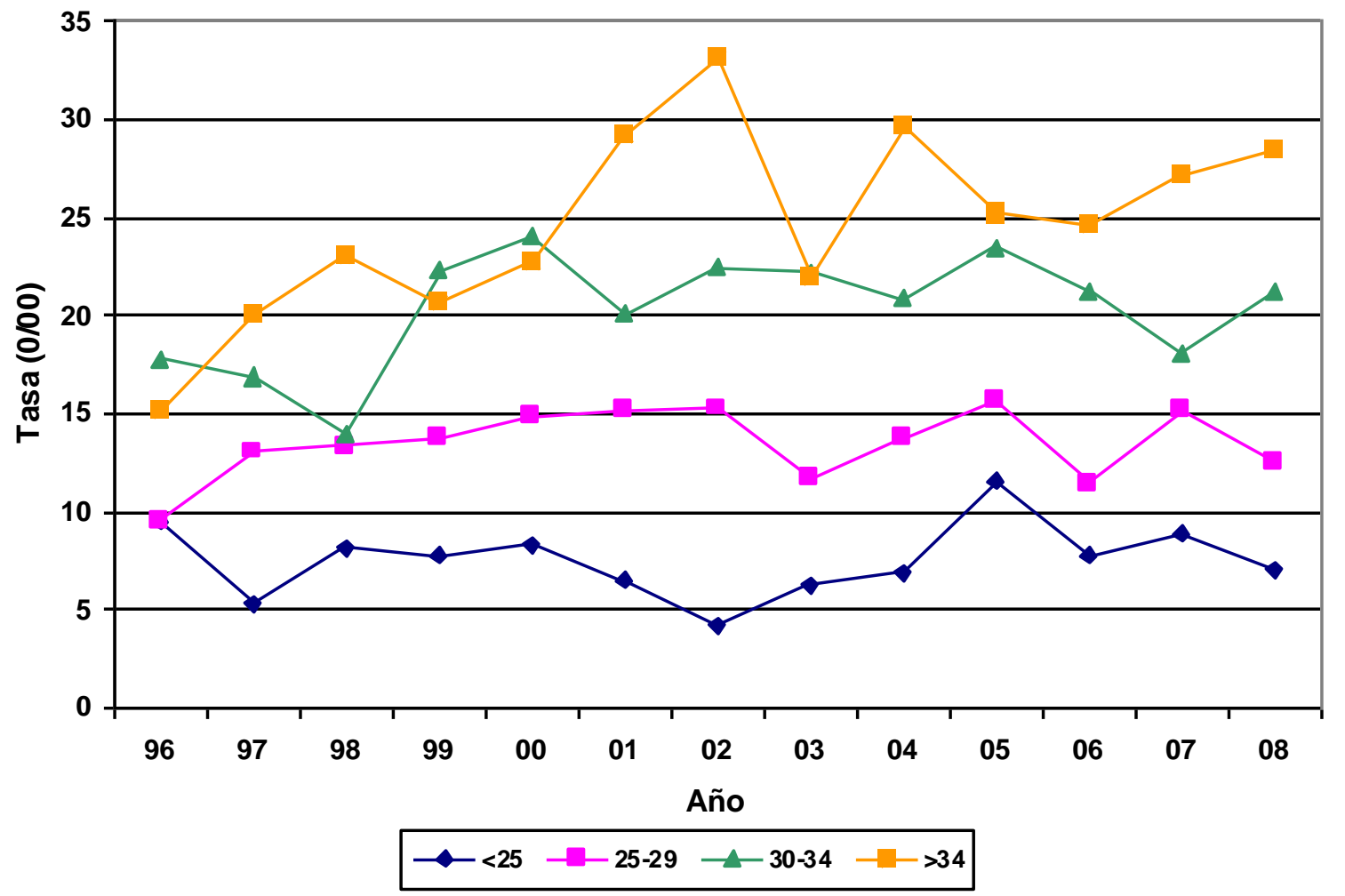

En el caso de la Región de Murcia, podemos encontrar claras similitudes con los datos de España, aunque también encontramos más irregularidades, lo que puede deberse en parte al menor tamaño de la población. Así, los grupos de mujeres mayores de 30 años, y especialmente mayores de 34 años, son también los que tienen mayores tasas de partos múltiples. En este caso, sin embargo, los datos no nos muestran el incremento constante y la estabilización que observábamos a nivel nacional. El grupo que tiene los valores más variables es el de mujeres de más de 34 años, con un importante incremento a partir del 2000 y un brusco descenso en la tasa del año 2003 que podrían estar relacionados con los cambios legislativos producidos en esas fechas.

\section{Edad media a la maternidad en la Región de Murcia y en España (1.996-2.007)}

En vista del incremento de partos múltiples observado en mujeres mayores de 30 años, se ha estudiado también la edad media de maternidad con objeto de analizar la tendencia en los últimos años y las posibles diferencias entre la Región y el conjunto de España.

En la Figura 5 se muestran los datos sobre la edad media a la primera maternidad. Como podemos observar, en ambos casos ha aumentado la edad media para el primer hijo, especialmente en el caso de Murcia. En España la media se ha incrementado en 0,98 años desde 1.996 hasta 2.007, y en Murcia en 1,55 años en el mismo período de tiempo, reduciéndose la diferencia que existía inicialmente. A pesar de ese mayor incremento en la Región de Murcia, la edad media para el primer hijo sigue siendo menor que en España, con alrededor de un año de diferencia. 
Figura 5: Edad media a la maternidad para el primer hijo en la Región de Murcia y en España (1.996-

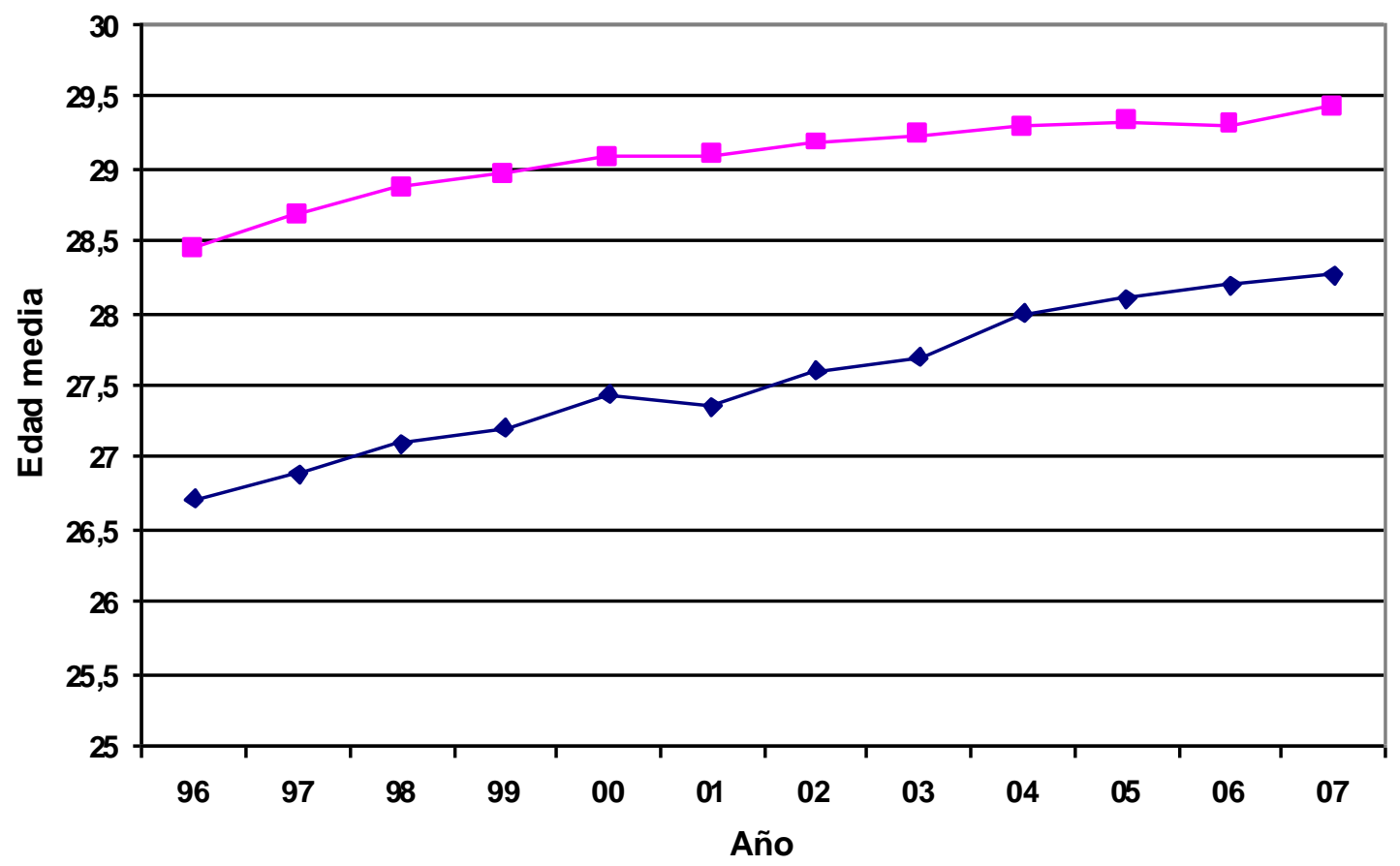

$\longrightarrow$ Murcia $\longrightarrow$ España

Por último, en la Figura 6 vemos la evolución seguida por la edad media a la maternidad (para todos los hijos, no únicamente para el primero). En este caso también se ha incrementado la edad media a nivel nacional y regional, pero el aumento ha sido mayor y más regular en el caso de España. Al igual que ocurría para el primer hijo, la edad media a la maternidad sigue siendo mayor en España que en Murcia pero, en este caso, las diferencias no muestran tendencia a disminuir, sino que se ampliaron a finales de los 90 y parecen haberse mantenido desde entonces. 
Figura 6: Edad media a la maternidad en la Región de Murcia y en España (1.996-2.007)

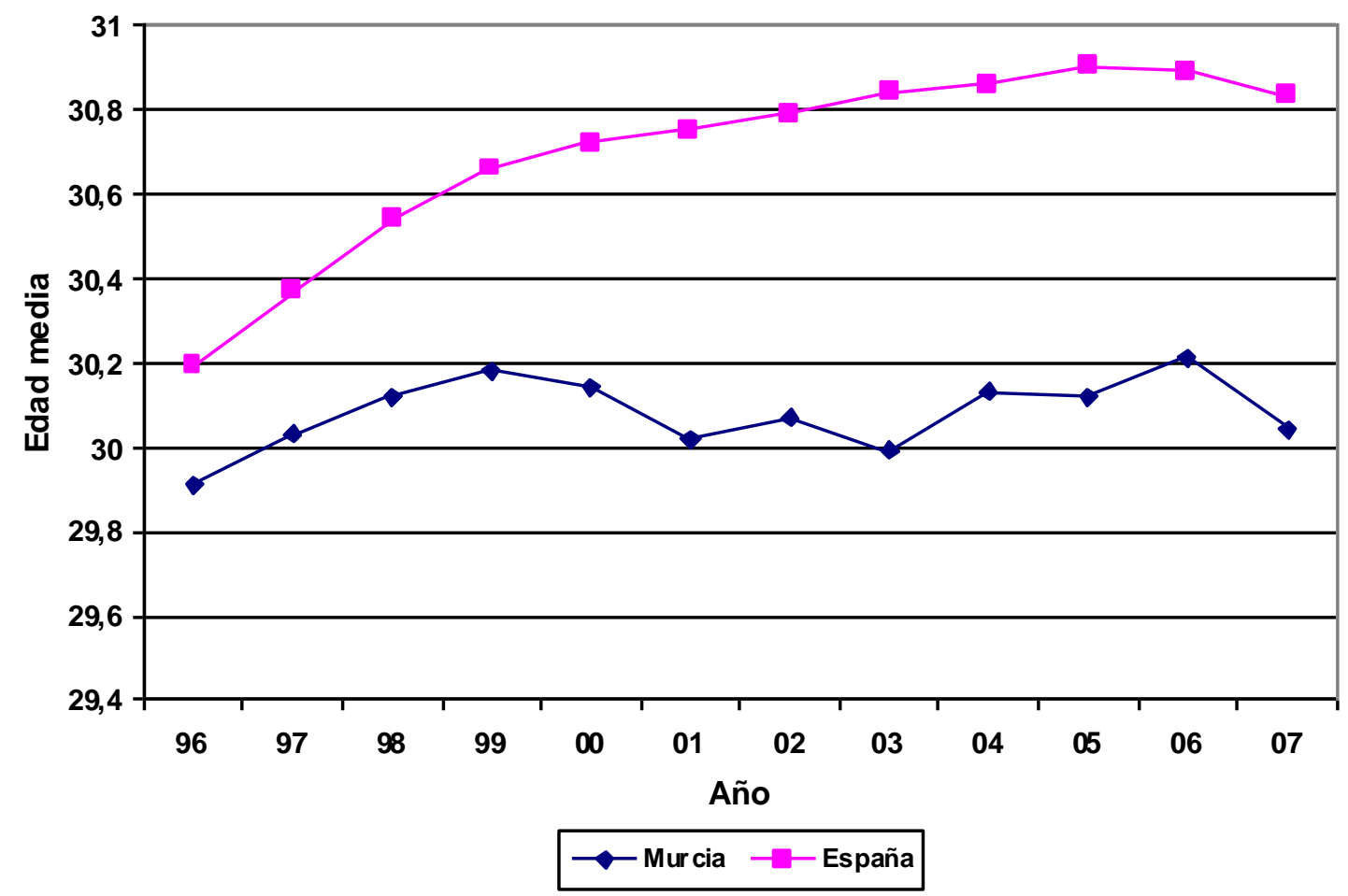

A la vista de estas gráficas no parece que el incremento global de la edad media a la maternidad tenga un efecto relevante sobre las tasas de partos múltiples que, si así fuera, deberían ser consistentemente mayores en España que en Murcia. Estas diferencias tampoco ayudan a explicar las variaciones anuales encontradas en la tasa, tanto para el conjunto como para grupos de edad específicos en la Región, que se podrían explicar mejor por el efecto de variaciones en la aplicación de las técnicas de reproducción asistida.

\section{CONCLUSIONES}

El número de partos múltiples ha aumentado de forma muy significativa en los últimos años tanto en la Región de Murcia como en España. La tasa de partos gemelares o de orden mayor ha pasado a ser de $13.1 \%$ en 1996 a 18.4\% en 2008 (12.7\% a 19.4\%o en España). Si tenemos en cuenta las tasas de partos múltiples según el grupo de edad de la madre, el mayor incremento se ha producido en los grupos de madres a partir de los 30 años; especialmente a partir de los 34. Esta tendencia de nuevo se repite en Murcia y en España, aunque a nivel nacional la variación es más regular, y a nivel regional los incrementos y descensos son más acusados.

Hay varios factores, compatibles entre sí, que pueden explicar esta variación. En primer lugar, este incremento ha estado aparejado a un aumento concomitante de nacimientos. Sin embargo este incremento no explica, por sí solo, la variación en partos múltiples, que ha sido mayor en términos relativos. Una segunda explicación podría estar en el aumento de la edad media a la maternidad. Como se ha visto, de acuerdo con los datos manejados, este incremento tampoco explicaría de forma convincente el importante crecimiento de la tasa de partos múltiples. Por otra parte, este aumento de la edad a la maternidad se relaciona 
también con la tercera, y más probable, explicación: el efecto de la creciente utilización de técnicas de reproducción asistida, especialmente en el grupo de mujeres de mayor edad ${ }^{1}$.

La tasa actual de partos múltiples, por otra parte, presenta una tendencia ligeramente ascendente 0 , en todo caso, hacia la estabilización. Es decir, no se vislumbra en la misma un descenso a corto o medio plazo. Esto implica que, probablemente, durante los próximos años seguiremos contando en España, y en la Región de Murcia en particular, con tasas cercanas al 20\%; es decir, alrededor de 350 partos múltiples anuales en la Región. Como hemos visto esto presenta algunas implicaciones importantes para el sistema de salud en su conjunto ${ }^{5}$ pero, sobre todo, tiene repercusiones decisivas en las familias y sus necesidades de adaptación a la nueva situación; adaptación a la que puede colaborar de forma decisiva el profesional sanitario proporcionando una información y orientación adecuadas.

Además, como hemos visto anteriormente, el deseo de tener un embarazo múltiple puede llevar a una subestimación de los riesgos, no sólo biomédicos sino también sociosanitarios y psicológicos, asociados a un embarazo de este tipo. Esto nos muestra la necesidad de dar una adecuada orientación y educación a los padres que se encuentran en estas situaciones. Esta orientación puede comenzar por los profesionales con los que tratan en la clínica de reproducción asistida (en el caso de un tratamiento de fertilidad), puede seguir con la asistencia durante el proceso del embarazo, y también incluye a los profesionales que asisten a la mujer durante el parto y los que se encargan después de asegurar la salud de los niños tras el nacimiento.

\section{REFERENCIAS}

1. Fuster, V., Zuluaga, P., Colantonio, S., y De Blas, C. (2008). Factors Associated with Recent Increase of Multiple Births in Spain. Twin Research and Human Genetics, 11 (1), 70-76.

2. Bruna Catalán, I., Pérez Milán, F., Tur Padró, R., Ricciarelli, E., De La Fuente Hernández, A., Monzó Miralles, A., Martínez-Salazar, J., Fernández-Shaw, S., Ardoy Vilches, M., Torelló Ibáñez, M. J., y Vila Álvarez, J. (2005). Multiple pregnancy after FIV-ICSI in Spain: incidence and criteria on the transference of embryos. Revista Iberoamericana de Fertilidad, 22, 99-110.

3. Leonard, L., Denton, J. (2006). Preparation for parenting multiple birth children. Early Human Development, 82, 371-378.

4. Campbell, D., Van Teijlingen, E., y Yip, L. (2004). Economic and social implications of multiple birth. Best Practice and Research Clinical Obstetrics and Gynecology, 18 (4), 657-668.

5. Prieto, B. (2005). Economic implications of multiple delivery. Revista Iberoamericana de Fertilidad, 22(5), 331-338.

6. Topp M, Huusom LD, Langhoff-Roos J, Delhumeau C, Hutton JL, Dolk H. (2004). Multiple birth and cerebral palsy in Europe: a multicenter study. Acta Obstet Gynecol Scand 83: 548-553.

7. Bryan, E. (2005). Psychological aspects of prenatal diagnosis and the implications in multiple pregnancies. Prenatal Diagnosis, 25, 827-834.

8. Damato, E. (2000). Maternal-Fetal Attachment in Twin Pregnancies. JOGNN: Clinical Studies, 29 (6), 598-605.

9. Child, T., Henderson, A., y Lin Tan, S. (2004). The Desire for Multiple Pregnancy in Male and Female Infertility Patients. Human Reproductions, 19 (3), 558-561. 
10.Kalra, S., Milad, M., Klock, S., y Grobman, W. (2003). Infertility Patients and Their Partners: Differences in the Desire for Twins Gestations. Obstetrics and Gynecology, $102(1), 152-155$.

11.Leonard, L. (1998). Depression and Anxiety Disorders During Multiple Pregnancy and Parenthood. JOGNN: Clinical Issues, 27 (3), 329-337.

12.Fisher, J., y Stocky, A. (2003). Maternal Perinatal Mental Health and Multiple Births: Implications for Practice. Twin Research, 6 (6), 506-513).

13. Yokoyama, Y. (2003). Comparison of Child-rearing Problems Between Mothers with Multiple Children who Conceived after Infertility Treatment and Mothers with Multiple Children who Conceived Spontaneously. Twin Research, 6 (2), 89-96.

14. Misri, S. (1996). Anxiety and mood disorders in pregnancy. Paper presented at Supporting Women, Supporting Ourselves: A Conference on Reproductive Psychiatry, November 23, 1996. Vancouver, BC.

15.Robin, M., Corroyer, D., y Casati, I. (1996). Childcare patterns of mothers of twins during the first year. Journal of Child Psychology and Psychiatry, 37(4), 453-460.

16. Leonard, L. (2003). Breastfeeding Rights of Multiple Birth Families and Guidelines for Health Professionals. Twin research, 6 (1), 34-45.

17. Gibson, F., y McMahon, C. (2004). Parenting and child psychosocial development after infertility management. International Congress Series 1266, 351-358.

18.www.ine.es

19.www.carm.es/econet 